\title{
A Retrospective Study Evaluating the Efficacy of Identification and Management of Sepsis at a Western Cape Province District Level Hospital Internal Medicine Department, in Comparison to the Guidelines Stipulated in the Surviving Sepsis Campaign 2012
}

\author{
Raisa Bhikoo ${ }^{1 *}$, Sarah Versfeld ${ }^{1}$, Basson $V^{2}$ and Almero H Oosthuizen ${ }^{3}$ \\ ${ }^{1}$ Department of Internal Medicine, Karl Bremer Hospital, South Africa \\ ${ }^{2}$ Head of Internal Medicine, Karl Bremer Hospital, South Africa \\ ${ }^{3}$ Department of Emergency Medicine, Karl Bremer Hospital, South Africa
}

Submission: February 08, 2017; Published: July 13, 2017

*Corresponding author: Raisa Bhikoo, Medical officer in the Department of Internal Medicine, Karl Bremer Hospital, South Africa, Email: raisa3121@gmail.com

Abstract

Background: Currently there is little data on identification, management and outcomes of patients with sepsis in developing countries. Simple cost effective measures such as accurate identification of patients with sepsis and early antibiotic administration are achievable targets that are within reach without having to make use of unsustainable protocols constructed by developed countries.

Aim: The aim of our study is to assess the efficacy of clinicians at a district level hospital in the Western Cape at identifying and managing sepsis. Furthermore we will assess the outcome of patients in terms of in-hospital mortality and length of hospital stay given the above management.

Methods: A retrospective study design was applied when analyzing data from the routine burden of disease audit done on a 3 monthly basis at Karl Bremer Hospital.

Results: The total sample size obtained was 70 patients. A total of $18 / 70(26 \%)$ patients had an initial triage blood pressure indicative of sepsis induced hypotension however only $1 / 18(5.5 \%)$ of these patients received an initial crystalloid fluid bolus of $30 \mathrm{ml} / \mathrm{kg}$. The median time for antibiotic administration in septic shock was 4.65 hours. Further a positive delay in antibiotic administration $(p$ value $=0.0039)$ was demonstrated. The data showed $8 / 12(66 \%)$ of patients with septic shock received inappropriate amounts of fluids. The in-hospital mortality for sepsis was found to be $4 / 24$ (17\%), for severe sepsis $11 / 34$ (32\%) and a staggering 9/12(75\%) for septic shock.

Conclusion: The outcomes of the study concluded that the initial classification process and management of sepsis by our clinicians is flawed. This inevitably leads to an increase in in-hospital mortality.

\section{Background}

Sepsis, as defined by the Surviving Sepsis Campaign 2012 (SCC) [1] is the presence of a probable or documented infection together with systemic manifestations of an infection. In 2004 the World Health Organization (WHO) [2] listed three infective causes namely lower respiratory tract infection,

diarrheal disease and tuberculosis on their top 10 list for causes of death, which is similar to mortality reports documented in the 2013 Western Cape mortality profile [3]. Thus highlighting sepsis and its squeal; which ultimately is caused by an infection as major contributors to the local and global burden of disease? 
To date there is virtually no accurate data on incidence, prevalence or mortality rates for sepsis, severe sepsis and septic shock in the developing countries. Mortality rates have been reported to be as high as 30\% for sepsis, $40 \%$ for severe sepsis and $80 \%$ for septic shock [4-6] in developed countries. Worldwide septic shock is still the leading cause for death in intensive care units [5]. Data from developed countries show a continuous increase in the incidence of sepsis thus further emphasizing the need to review management protocols in order to reduce morbidity and mortality. In developed countries the implementation of protocols for the identification of sepsis and management thereof, have contributed to a decline in mortality rates [7].

In low income countries major concerns regarding accessibility to healthcare, limitations due to costs, lack of resources and delayed presentations of patients with sepsis make implementing such protocols, based on developed countries patients profile difficult. Thus in Sub-Saharan Africa there has been a widespread shift towards protocol development that is cost effective and specific for the epidemiologic and ecologic data [8,9]. In 2006 a Ugandan based prospective study assessed the management and outcomes of hospitalized patients with severe sepsis syndromes. Approximately $85 \%$ of their sample was HIV positive.

Factors contributing to mortality included inadequate fluid administration, lack if uniformity in source appropriate antibiotic administration and delay in antibiotic administration [8]. A follow-up prospective study was then done in 2008 using the previous study as the observation cohort. Interventions included early appropriate antibiotic and intravenous fluid administration. Mortality at 30 days was significantly lower in the intervention cohort compared to the observation cohort concluding that simple and inexpensive management could improve outcome $[8,9]$. The surviving sepsis campaign [1] is an initiative that initially was published in 2004 reviewing data on the management of severe sepsis and septic shock. The recommendations are intended to be best practice and by no means standard of care. Currently no data is available in South Africa regarding sepsis management in our unique setting thus we hope to pilot the way for further research in this field.

\section{Demographics}

Karl Bremer Hospital (KBH) is a large district level hospital situated in Bellville, Cape Town, with a total of 310 beds. The hospital services on average 11000 patients per month. The estimated total cost per day, per patient, is R2105.90 for a ward admission. Almost $72 \%$ of the patients are classified as either earning no income or have an average earning of less than R3000 per month, and hence are not obligated to pay for full medical fees. Furthermore there is a 4 bed high care unit which is shared by the all departments. The high care unit is able to manage ventilated patients however if a prolonged high care admission is anticipated, these patients would need to be transferred to a fully equipped ICU at a tertiary facility such as Tygerberg Hospital that is managed by a dedicated ICU team. Additionally the emergency department (ED) has 3 resuscitation beds for all emergencies that may present to the casualty.

\section{Methodology}

The primary aim of our study was to determine the efficacy of sepsis identification and management by clinicians at Karl Bremer Hospital. Furthermore we aimed to determine the demographics of patients presenting with sepsis, and also the burden of sepsis and its management, on the mortality rates and length of hospital stay amongst patients admitted. Key determinants of efficacy were assessed using guidelines outlined in the Surviving Sepsis Campaign 2012 as a means of comparison. A retrospective descriptive study design was performed in order to achieve the above. Ethics approval was obtained from the Health Research Ethics Committee at the University of Stellenbosch. The internal medicine department performs a routine burden of disease audit on a three monthly basis. Data for the audit is obtained by means of triplicate discharge letters, hospital transfer notes and death certification summaries containing all relevant information of the patients hospital stay from admission to discharge or death.

Data from the burden of disease audit completed in October 2015 and January 2016 was reviewed for patient selection. In order to select patients for the study the data from the burden of disease audit sheets had to state any one of the following key words namely sepsis, severe sepsis or septic shockas the diagnosis at discharge or death. The suspected or confirmed infection had to be present at emergency department presentation in order to qualify for the study. Data was assessed using a data collection sheet compiled by the authors that focused specifically on clinical and blood gas measures that could be used in the emergency department to classify patients as sepsis, severe sepsis and septic shock. Time to first dose antibiotics (grade 1B evidence based on SCC), source appropriate antibiotics (grade 1B), amount of intravenous fluids (grade 1c) and arterial blood gas (ABG)/lactate measurement was also assessed.

Once the data was obtained we retrospectively classified patients into either sepsis, severe sepsis or septic shock based on information that was available to the casualty doctor at the time of presentation. All data collected was captured onto a Microsoft Excel@ database. Data analysis was done in Microsoft Excel@ and statistical analysis in Statistica(C). Statistical significance was considered if the $\mathrm{p}$ value $<0.05$. To compare descriptive variables the sign test was used. To assess for data association logistic regression testing and negative binomial regression testing was performed. Here the data was interpreted as odds ratios (OR) with a $95 \%$ confidence interval (Table 1). 
Table 1: Definitions used for Sepsis Classification.

\section{Sepsis}

Wide spread systemic manifestations due to the existence of an infection. The systemic manifestations could be any of the following: (adapted from the SSC 2012)[1]

General variables

- $\quad$ Fever $\left(>38.3^{\circ} \mathrm{C}\right)$

- $\quad$ Hypothermia (core temperature $<36^{\circ} \mathrm{C}$ )

- Heart rate $>90 / \mathrm{min}$

- Tachypnea, RR $>25$

- $\quad$ Altered mental status

- $\quad$ Significant edema or positive fluid balance $(>20 \mathrm{~mL} / \mathrm{kg}$ over $24 \mathrm{hr})$

- Hyperglycemia (plasma glucose $>140 \mathrm{mg} / \mathrm{dL}$ or $7.7 \mathrm{mmol} / \mathrm{L}$ ) in the absence of diabetes

Inflammatory variables

- Leukocytosis $(\mathrm{WBC}>10)$ or Leukopenia $(\mathrm{WBC}<4)$

- $\quad$ Plasma CRP > 4

Hemodynamic variables

- $\quad$ Arterial hypotension ( $\mathrm{SBP}<90 \mathrm{~mm} \mathrm{Hg}, \mathrm{MAP}<70 \mathrm{~mm} \mathrm{Hg}$ )

Organ dysfunction variables

- $\quad$ Arterial hypoxemia (Pao2/Fio2 $<300$, PF ratio)

- Acute oliguria (urine output $<0.5 \mathrm{~mL} / \mathrm{kg} / \mathrm{hr}$ for at least $2 \mathrm{hrs}$ despite adequate fluid resuscitation)

- $\quad$ Creatinine increase $>44.2 \mathrm{micromol} / \mathrm{l}$

- Coagulation abnormalities (INR $>1.5$ or aPTT $>60 \mathrm{~s}$ )

- $\quad$ Ileus (absent bowel sounds)

- $\quad$ Thrombocytopenia (platelet count < 100)

- Hyperbilirubinemia (plasma total bilirubin $>4 \mathrm{mg} / \mathrm{dL}$ )

Tissue perfusion variables

- $\quad$ Hyperlactatemia (> $1 \mathrm{mmol} / \mathrm{L})$

- Decreased capillary refill or mottling

II. Severe Sepsis

Sepsis PLUS acute organ dysfunction or tissue hypoperfusion:

Sepsis-induced hypotension:

- a systolic blood pressure(SBP) $<90 \mathrm{~mm} \mathrm{Hg}$, or

- mean arterial pressure (MAP) $<70 \mathrm{mmHg}$, or

- $\quad$ SBP decrease $>40 \mathrm{~mm} \mathrm{Hg}$

Organ dysfunction variables

- $\quad$ Arterial hypoxemia $(\mathrm{Pao} 2 / \mathrm{Fio} 2<300)$

- $\quad$ Acute oliguria (urine output $<0.5 \mathrm{~mL} / \mathrm{kg} / \mathrm{hr}$ for at least $2 \mathrm{hrs}$ despite adequate fluid resuscitation)

- $\quad$ Creatinine increase $>45$

- Coagulation abnormalities (INR $>1.5$ or aPTT $>60 \mathrm{~s}$ )

- $\quad$ Ileus (absent bowel sounds)

- Thrombocytopenia (platelet count $<100$ )

- $\quad$ Hyperbilirubinemia (plasma total bilirubin $>4 \mathrm{mg} / \mathrm{dL}$ ) 
Tissue perfusion variables

- $\quad$ Hyperlactatemia $(>1 \mathrm{mmol} / \mathrm{L})$

- Decreased capillary refill or mottling

III. Septic Shock

Severe sepsis PLUS evidence of tissue hypoperfusion not reversed with effective fluid resuscitation.

\section{Sepsis-induced tissue hypoperfusion:}

Blood lactate concentration $>4 \mathrm{mmol} / \mathrm{L}$

OR

\section{Sepsis-induced hypotension: as above}

\section{Statistical Results}

From the 1000 patients reviewed in the burden of disease audit, 70 patients $(7 \%)$ were included in the study. Of the 70 patients 34 were male (48.5\%) and 36 were female (51.4\%). The overall mean age of patients presenting with a sepsis syndrome was $48(\mathrm{SD} \pm 9.5, \min =17, \max =85)$. A total of 25 patients $(36 \%)$ were HIV positive. The most common infective cause for sepsis across all spectrums identified by emergency personnel was a lower respiratory tract infection. Not surprisingly diarrheal disease/acute gastroenteritis was the second most common diagnosis made (Figure 1, 2 \& 3).

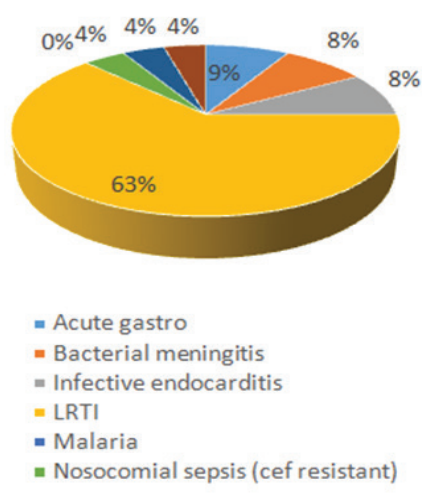

Figure 1: Suspected source for sepsis.

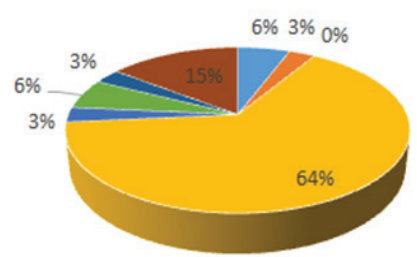

$$
\begin{array}{ll}
\text { " Acute gastro } & \text { = Bacterial meningitis } \\
\text { " Infective endocarditis } & \text { " LRTI } \\
\text { - Malaria } & \text { " Nosocomial sepsis (cef resistant) } \\
\text { - Unknown source } & \text { "UTI }
\end{array}
$$

Figure 2: Suspected source for severs sepsis.

Based on the data collection and subsequent sepsis sub grouping, evaluated using the initial information available to the casualty doctor, $24 / 70$ patients (34\%) were classified as having sepsis, 34/70 (48\%) had severe sepsis and 12/70 (17\%) had septic shock. However 18/24 (75\%) of patients with sepsis, $8 / 34(25 \%)$ of patients with severe sepsis and $3 / 12(25 \%)$ of patients with septic shock had no arterial blood gas or lactate measured at initial presentation. No patients who had an initial lactate measured then had a repeat lactate measured in order to assess for lactate clearance or fluid responsiveness. Thus as a result many of the patients who likely should have been classified as septic shock were then classified as severe sepsis based on adherence to definitions. A total of $18 / 70$ (26\%) patients had an initial triage blood pressure indicative of sepsis induced hypotension however only 1/18 (5.5\%) of these patients received an initial crystalloid fluid bolus of $30 \mathrm{ml} /$ $\mathrm{kg}$ and subsequent immediate blood pressure recheck for the fluid responsive status. Furthermore 6/18 (33\%) patients had no initial arterial blood gas or lactate measured, thus authors had no way of knowing whether the initial classification of the patient was severe sepsis or septic shock, forcing us to assign patients to the severe sepsis group.

$\begin{aligned} & \\ & =\text { Acute gastro } \\ & \text { = Bacterial meningitis } \\ & \text { = Infective endocarditis } \\ & =\text { - LRTI } \\ & \text { - Malaria }\end{aligned}$

The most frequently prescribed antibiotic across all categories for sepsis was ceftriaxone (Figure 4). Bearing in mind the above literature review regarding source appropriate antibiotics $55 / 70$ patients $(78.5 \%)$ were deemed to have received the correct antibiotics prescribed for the respective source. However no HIV positive patient included in the study received cover for mycobacterium tuberculosis or pneumocystis jirovecii pneumonia in the first 24 hours of presentation, despite the clinician having a documented suspicion thereof. 


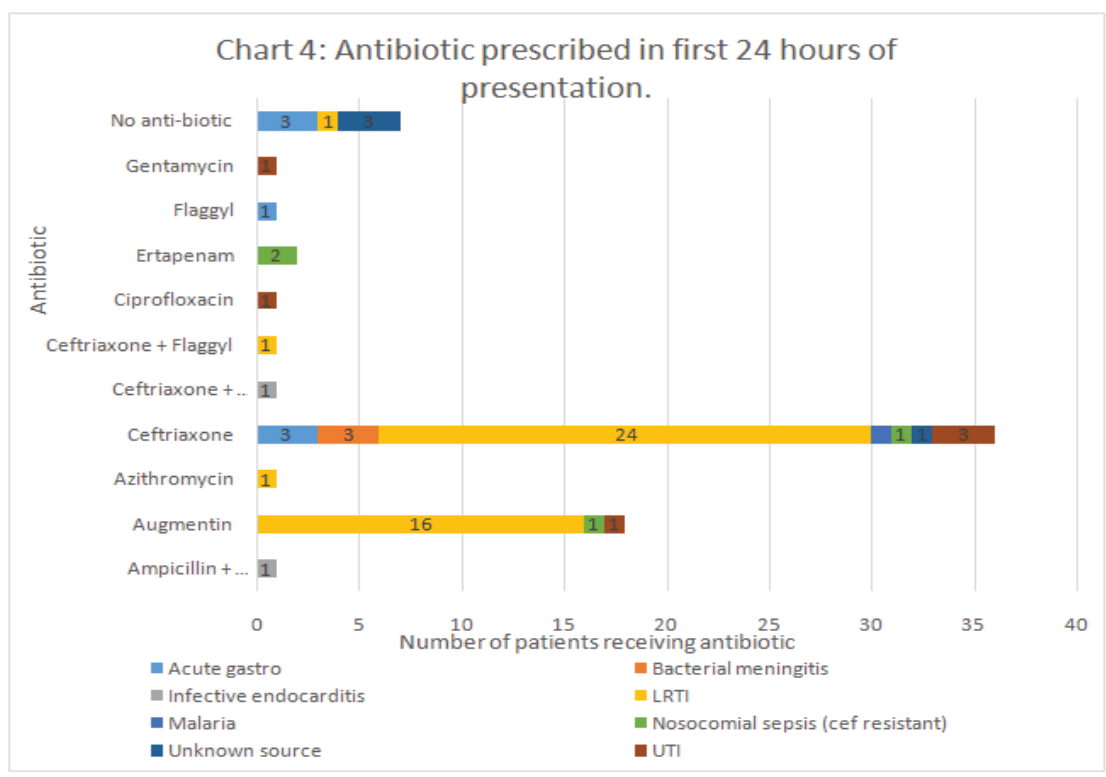

Figure 4: Antibiotic prescribed in first 24 hours of presentation.

Interestingly, what is also important to note is that $7 / 70(10 \%)$ of the patients received no antibiotics in the first 24hours of presentation to hospital despite being identified by the casualty doctor as having a possible infective cause for clinical symptomatology. Here the likely source was equally distributed between acute gastroenteritis and an unknown source for sepsis. Of these patients $3 / 7$ of the patients could be classified as having septic shock based on initial lactate measurement. The median time for antibiotic administration in the first 24 hours of presentation across all subgroups included 3.63 hours for sepsis (range 1.67-10.30, $\max =24$ hours), 1.58 hours for severe sepsis (range 1.00-2.83, max 24hours) and 4.25 hours for septic shock (range 1.00-23.98, max 24 hours). What is very important to note here is that this is the time taken from the attending doctors consult to actual antibiotic administration. Hence the time from initial presentation to the emergency department to actual antibiotic given may differ depending on waiting times. This unfortunately could not be assessed due to lack of documentation of initial presenting time.

In order to assess whether antibiotics were given within the 3 hour time frame for sepsis and severe sepsis the sign test was used to extrapolate the data. The results showed that for both sepsis and severe sepsis there was no delay in antibiotic administration ( $p$ value $=0.2706$ for sepsis and 0.9997 for severe sepsis), bearing in mind that the 3 hour mark was used as the cut off for optimal time to initial antibiotic administration. For septic shock however a cut off of 1 hour was used for optimal time to antibiotic administration, here the sign test showed a positive delay in antibiotic administration ( $p$ value $=0.0039$ ).

Regarding the early appropriate intravenous fluid administration discussed above, the data showed that 5/24
(20\%) of patients with sepsis, 8/34 (23\%) of patients with severe sepsis and 8/12 (66\%) of patients with septic shock received inappropriate amounts of fluids. As mentioned before only 1 patient received an initial fluid bolus of $>30 \mathrm{ml} / \mathrm{kg}$ for a sepsis induced hypotension. Using the Pearson chi-square test a positive association between the patients with septic shock and inappropriate intravenous fluid administration was noted ( $\mathrm{P}$ value $=0.009$ ). Furthermore the proportion of septic shock patients that received appropriate intravenous fluids was less than half that of the sepsis and severe sepsis group. As mentioned no patients in our study had repeat ABG'S done in order to assess for lactate clearance, a marker of response to therapy. Also no invasive measures such as central venous pressures or arterial line blood pressure readings were used to optimally assess fluid responsiveness in the first 24 hours of management.

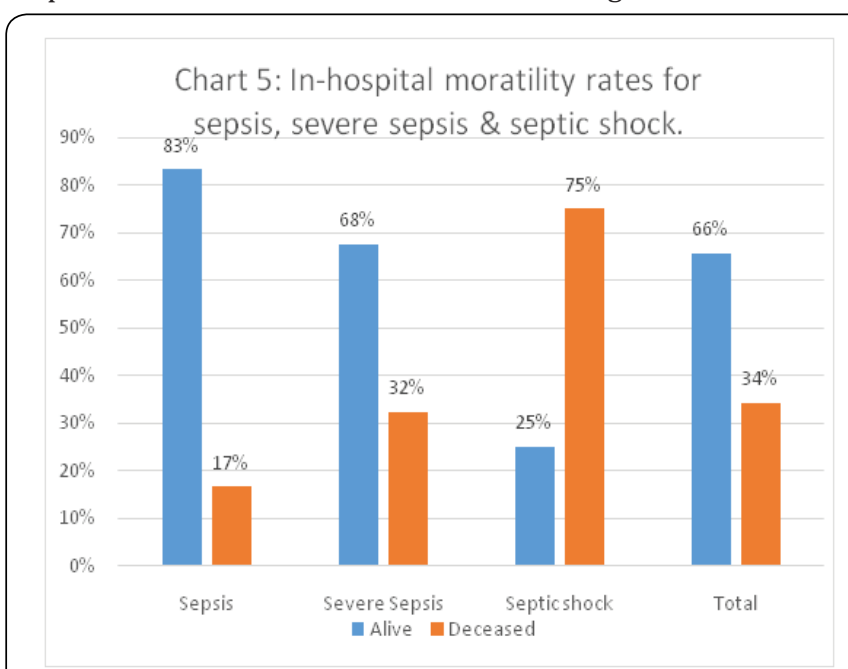

Figure 5: In-hospital morality rates for sepsis, severe sepsis \& septic shock. 
Overall the outcome of patients in the study was determined by either discharge or in-hospital mortality. The in- hospital for mortality for sepsis being $4 / 24$ (17\%), for severe sepsis $11 / 34$ (32\%) and a staggering $9 / 12(75 \%)$ for septic shock (Figure 5). The mean age of deceased patients across all sepsis syndromes is 53 ( $\mathrm{SD} \pm$ 9.5). Furthermore logistic regression testing was done in order to investigate for a possible association between in- hospital mortality rates and various sepsis management principles. A positive association between in-hospital mortality and the following was found:

1. Time to first dose antibiotic administration $(\mathrm{OR}=1.07$, $\mathrm{P}$ value $=0.027,95 \% \mathrm{CI}=1.008-1.14)$. For every 1 hour delay in antibiotic administration the chance of death increased by $7 \%$.

2. Source appropriate antibiotics $(\mathrm{OR}=0.17, \mathrm{P}$ value $=$ $0.005,95 \% \mathrm{CI}=0.048-0.59$ ). The chance of death amongst patients that received source appropriate antibiotics is $83 \%$ less than those who did not.

3. Early appropriate intravenous fluid administration $(\mathrm{OR}=0.33$, $\mathrm{P}$ value $=0.040,95 \% \mathrm{CI}=0.11-0.95)$. An appropriate intravenous fluid was associated with a $67 \%$ reduction in inhospital mortality.

The mean length of stay for all sepsis syndromes was 6.3 days ( $\mathrm{SD} \pm 2.3$ days).

\section{Discussion}

The statistics above depict clear faults in the identification of sepsis and its resultant suboptimal management. Evidence to support the poor recognition of sepsis syndromes is noted by the lack of adequate fluid boluses for patients that met the definition for hypotension at admission. This indicates that clinicians lack the understanding of hypotension as a clinical indicator of organ dysfunction in sepsis. Clinicians at Karl Bremer Hospital do not have access to laboratory results for a minimum of 12 hours post consult and are thus forced to make use of clinical judgment regarding severity of disease. This can be aided by blood pressure monitoring, urine output and blood gas measurement for lactate and PF ratios to determine fluid responsiveness and organ dysfunction. Further data supporting inadequacies in sepsis identification is evidenced by the $3 / 7$ patients, that could be classified as septic shock based on the initial blood gas lactate measurement, who did not receive antibiotics for the first 24 hours after presentation. This indicates a poor understanding of the effect of delayed antibiotics on mortality rates related to sepsis. The overall median time to initial antibiotic administration was 4.25 hours in septic shock versus 1.58 hours in severe sepsis. Because these 3 patients were patients with septic shock it significantly increased the median time to initial antibiotic administration in this subgroup.

Kumar, et al. [10] showed a decrease in survival by 7.6\% for every 1 hour delay in antibiotic therapy over the ensuing 6 hours.
Comparatively our study showed a 7\% increase in mortality for every hour delay in antibiotic administration. As mentioned median time to first dose antibiotics was assessed from time of doctor/patient consult to initial antibiotic administration. The minimum waiting time in the ED from arrival at the hospital to doctor consult is $45-60 \mathrm{~min}$. Thus regardless of the difference neither group is meeting the target 3 hour and 1 hour administration time frame. This can be attributed to both poor identification as discussed above and resource limitations. The nursing staffs in the ED who are responsible for drug and fluid administration often oversee the management of 10-20 patients at any given moment. This makes it difficult for them to perform important tasks in a timorously manner. Bed constraints and lack of resuscitation room availability mean that severely ill patients will often lie in the general ED area where there is no appropriate monitoring.

Regarding source appropriate antibiotics a positive association with mortality was shown in our study. Leibovici $L$, et al. [11] showed improved survival when empiric antibiotic treatment matched the in vitro susceptibility of the likely pathogen. The concern with this is that we have limited evidence regarding source appropriate antibiotics in a HIV prevalent society. Infective etiologies in sub -Saharan Africa differ from those reported in the surviving sepsis guidelines; in fact certain studies reviewed by the committee excluded HIV positive patients. Begging the question which is the early source appropriate antibiotics that should be administered given the unique sub-Saharan Africa ecology and what is the effect on outcome in HIV positive patients.

Appropriate intravenous fluid administration is a further area for debate in the HIV prevalent South African setting. Both studies done in Uganda and Zambia [12] reported concerns regarding large fluid boluses with a resultant worsening respiratory failure in this setting. Despite this our findings show a $67 \%$ reduction in in-hospital mortality with appropriate intravenous fluid administration. Again concerns regarding appropriate monitoring in the emergency department make appropriate fluid administration difficult, patients receiving large fluid boluses run the risk of becoming fluid overloaded unless adequately monitored.

\section{Recommendations}

It is evident from the above that education for all health care providers involved in sepsis identification and management is necessary. A suggestion for process formation perhaps in the form of a sepsis check sheet may lead to improved management. Re-evaluation of outcomes in the form of length of hospital stay and in-hospital mortality will need to done in order to evaluate the impact of process formation. Further areas that need attention is improved availability of early laboratory results which aids decision making. Also increasing physician availability to decrease ED waiting times. 


\section{Conclusion}

The primary aim of our study was to evaluate the identification and management of the sepsis syndromes at a district level hospital in the Western Cape Province. The outcomes of the study concluded that the initial classification of sepsis, severe sepsis and septic shock by our clinicians is flawed. This is largely due to a lack of understanding by medical personnel regarding the clinical evidence needed to support the classification process. This clinical evidence is not dependent on delayed laboratory results, but can be found on basic clinical assessment and investigations available in the emergency department.

Priorities in the management of the sepsis syndromes that have been shown in our study to negatively effect in-hospital mortality include early source appropriate antibiotics and early appropriate intravenous fluid administration. These should be the cornerstones of management and can be instituted regardless of resource availability. In conclusion it is evident that additional research is needed in the field of sepsis identification and management in a resource limited setting, however basic management principles can still be implemented with the potential for an inordinate impact on patient survival.

\section{Acknowledgement}

A special thank you to Dr Zirkia Joubert and all the internal medicine medical officers at Karl Bremer Hospital, for granting us the time we needed to work on this study as well as for all their assistance with patient identification and data collection. We would like to thank Mr. MT Chirehwa for the data analytics and his subsequent interpretation of the statistical outcomes. Finally, we would like to thank Iesrafeel Jakoet and Brendon Versfeld for all their help with the write-up and editing of our study.

\section{Conflict of interest}

The authors have no conflict of interest to report. The project was funded solely by the named authors below.

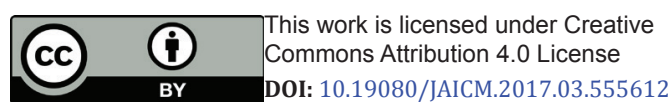

\section{References}

1. Dellinger RP, Levy MM, Rhodes A, Annane D, Gerlach H, et al. (2012) Surviving sepsis campaign: international guidelines for management of severe sepsis and septic shock. Crit Care Med 41(2): 580-637.

2. World Health Organization (2004) The Global Burden of Disease: 2004 update.

3. Government. Western Cape Mortality profile 2013.

4. Catenacci MH, King K (2008) Severe sepsis and septic shock: improving outcomes in the emergency department. Emerg Med Clin North Am 26(3): 603-623.

5. Degoricija V, Sharma M, Legac A, Gradiser M, Sefer S, et al. (2006) Survival analysis of 314 episodes of sepsis in medical intensive care unit in university hospital: impact of intensive care unit performance and antimicrobial therapy. Croat Med J 47(3): 385-397.

6. Kumar G, Kumar N, Taneja A, Kaleekal T, Tarima S, et al. (2011) Nationwide trends of severe sepsis in the 21st century (2000-2007). Chest 2011 140(5): 1223-1231.

7. Kaukonen KM, Bailey M, Suzuki S, Pilcher D, Bellomo R (2014) Mortality Related to Severe Sepsis and Septic Shock Among Critically Ill Patients in Australia and New Zealand, 2000-2012. JAMA 311(13): 1308-1316.

8. Jacob ST, Banura P, Baeten JM, Moore CC, Meya D, et al. (2012) The impact of early monitored management on survival in hospitalized adult Ugandan patients with severe sepsis: a prospective intervention study. Crit Care Med 40(7): 2050-2058.

9. Jacob ST, Moore CC, Banura P, Pinkerton R, Meya D, et al. (2009) Severe sepsis in two Ugandan hospitals: a prospective observational study of management and outcomes in a predominantly HIV-1 infected population. PLoS One 4(11).

10. Funk D, Kumar A (2006) Antimicrobial Therapy for Life-Threatening Infections: Speed is Life. Crit Care Med 34: 1589-1596.

11. Leibovici L, Shraga I, Drucker M, Konigsberger H, Samra Z, et al. (1998) The benefit of appropriate empirical antibiotic treatment in patients with bloodstream infection. J Intern Med 244(5): 379-386.

12. Andrews B, Muchemwa L, Kelly P, Lakhi S, Heimburger DC, et al. (2014) Simplified severe sepsis protocol: a randomized controlled trial of modified early goal-directed therapy in Zambia. Crit Care Med 42(11): 2315-2324.

\section{Your next submission with Juniper Publishers} will reach you the below assets

- Quality Editorial service

- Swift Peer Review

- Reprints availability

- E-prints Service

- Manuscript Podcast for convenient understanding

- Global attainment for your research

- Manuscript accessibility in different formats

( Pdf, E-pub, Full Text, Audio)

- Unceasing customer service

Track the below URL for one-step submission https://juniperpublishers.com/online-submission.php 